\title{
High-throughput detection
} of antioxidants in mulberry fruit using correlations between high-resolution mass and activity profiles of chromatographic fractions

Ye Ji Park ${ }^{1,2}$, Si Hyun Seong ${ }^{1,3}$, Min Sun Kim', Sang Wan Seo ${ }^{4}$, Mee Ree Kim² ${ }^{2^{*}}$ and Hyun Sik Kimº

\begin{abstract}
Background: Plant extracts contain a huge variety of pharmacologically active substances. Conventionally, various chromatographic methods must be applied several times to purify functional compounds to measure their functional activity. However, conventional purification methods are time-consuming and expensive due to the laborious purification process. Recently, a high-throughput discovery method that replaces such time-consuming purification processes was introduced; this method uses $15 \mathrm{~T}$ ultra-high-resolution Fourier transform ion cyclotron resonance mass spectrometry (15 T FT-ICR MS) and a high-throughput screening method. This 15 T FT-ICR MS provides unparalleled resolution and sub-ppm accuracy in mass measurements, while simultaneously detecting multiple compounds without separation. The high-throughput, simultaneous multi-component discovery method known as Scaling of Correlations between Activity and Mass Profiles (SCAMP) was used to detect functional compounds in a plant extract. We validated the performance of SCAMP using 33 fractions from antioxidant-rich mulberry ethyl acetate extract and known standard antioxidants.

Results: The mulberry fruit was first separated into 33 fractions by LC and analyzed using high-resolution mass spectrometry. The antioxidative strength of the 33 fractions and standard antioxidants was measured. To validate the efficiency of this antioxidant discovery method, correlations between the antioxidation activity profile and changes in mass intensity of components within the 33 fractions were calculated to provide relative scores for the antioxidant candidate list. Enrichment curves and area under the curve (AUC) values were then calculated to compare the performance of the methods. Using this improved scoring method, five strong antioxidants, chlorogenic acid (14.2 ng), dihydoxy quercetin (46.2 ng), rutin (154.0 ng), quercetin (71.7 ng) and luteolin (3.5 ng) in 2 kg mulberry fruit, were found within the top 20 candidates.
\end{abstract}

Conclusions: We calculated AUCs in order to compare scoring methods quantitatively. Scoring systems were compared and calculated AUCs, where the AUCs for new scoring systems (0.98 and 0.99) were higher than the previously used correlation coefficient ( $A \cup C=0.89$ ). Using the new scoring algorithms, we successfully enriched thirteen unknown strong antioxidant candidates in addition to known antioxidants, methyl syringin and naringenin (3.5 ng)

\footnotetext{
*Correspondence: mrkim@cnu.ac.kr; fticr@kbsi.re.kr

1 Mass Spectrometry and Advanced Instrumentation Group, Korea Basic

Science Institute, Cheongju, Chungcheongbuk-do 28119, Korea

${ }^{2}$ College of Human Ecology, Chungnam National University,

Daejeon 34134, Korea

Full list of author information is available at the end of the article
} 
in mulberry extract. Targeted purification of these unknown candidates will significantly reduce purification time and labor.

Keywords: Morus alba, FT-ICR MS, Isotopic fine structure, Molecular formula determination, SCAMP, DPPH, Scoring, Enrichment, AUC, Discovery, Functional molecule

\section{Background}

Plant extracts contain a huge number of pharmacologically active substances [1-3]. Conventionally, various preparative chromatographic methods are applied several times to purify functional compounds in order to estimate their functional activity. However, conventional purification methods [4] are time-consuming, complex, and expensive due to the purification process necessary prior to activity assays of functional components. Recently, a high-throughput multi-component discovery method that does not need time-consuming purification steps was introduced [5]; this method combines $15 \mathrm{~T}$ ultra-high-resolution Fourier transform ion cyclotron resonance mass spectrometry (15 T FT-ICR MS) and high-throughput screening. Ultra-high-resolution Fourier transform ion cyclotron resonance mass spectrometry (UHR FT-ICR MS) provides unparalleled resolution and sub-ppm accuracy in mass measurements [6-8], while simultaneously detecting multiple ions in a mixture without separation. UHR FT-ICR MS can be used to determine the molecular formula of small organic molecules (less than $500 \mathrm{amu}$ ) if the molecular mass is measured at $1 \mathrm{ppm}$ accuracy alongside its isotopic pattern [9]. UHR FT-ICR MS can be used to determine isotopic fine structure (IFS) and thus the molecular formula for small organic compounds $[10,11]$. If the relative activity of sequentially separated chromatographic fractions can be measured with high-throughput screening, and if the mass intensity for a given compound shows a similar trend to that of the total activity change over all fractions (as shown in Fig. 1), then that compound may contribute to the total activity of the fraction. A high-throughput simultaneous multi-component discovery method, known as Scaling of Correlations between Activity and Mass Profiles (SCAMP), was proposed to discover functional compounds in plant extracts; this method uses the correlation between mass intensity profiles and activity profiles of sequential fractions [5]. If the relative correlation coefficient between mass intensities and activity profiles can be calculated, active candidates and their relative activities can be quickly identified. However,

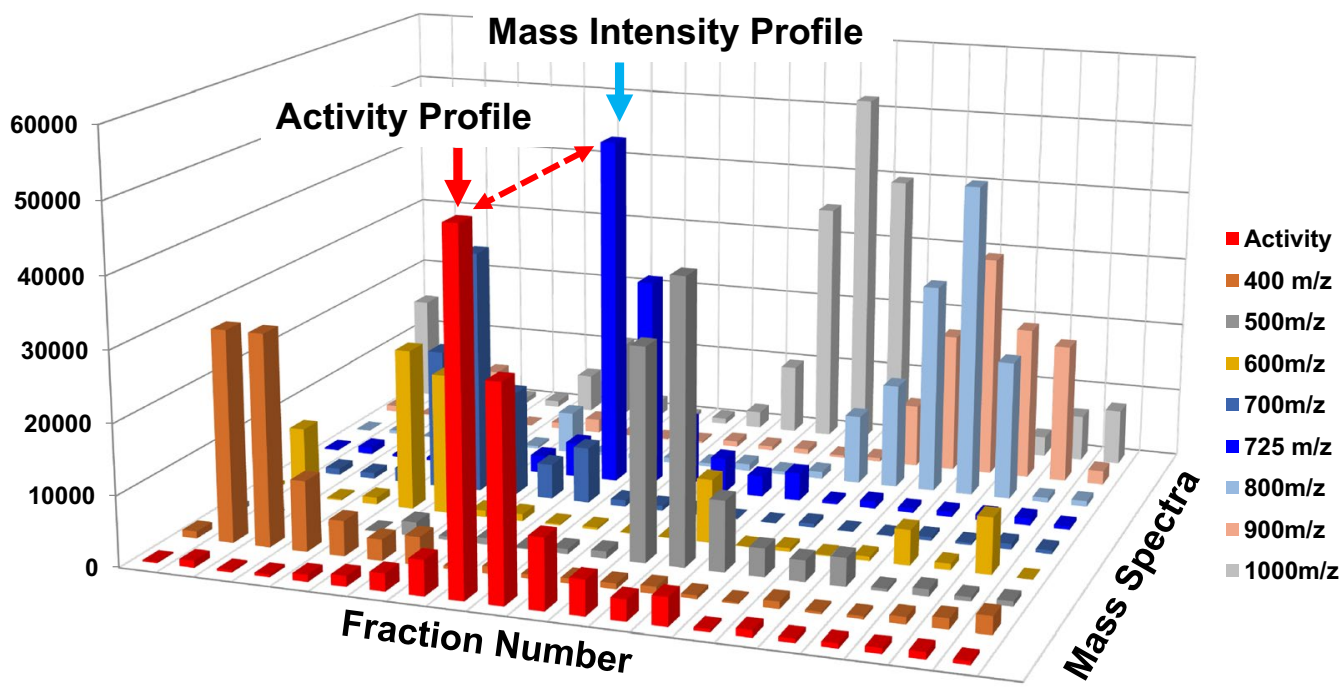

Fig. 1 A conceptual representation of the Scaling of Correlations between Activity and Mass Profiles (SCAMP) method. If the relative activity of sequentially separated chromatographic fractions can be measured and the mass intensity for a given compound shows a similar trend to that of the total activity change over all fractions, then that compound may contribute to the total activity 
preliminary results from early stages of SCAMP have included only a few fractions (specifically, 11 fractions) to show correlation behavior statistically [5]. Thus, we measured antioxidation activity and acquired UHR mass spectra using 33 fractions from antioxidantrich mulberry ethyl acetate extract (EAEM), as well as known standard antioxidant solutions (SAOx). All the standard antioxidants are selected from the published antioxidant list [12-18]. SCAMP performance was validated using UHR MS and activity data by listing strong antioxidant candidates. SAOx will likely be listed within the high score region due to high correlation scores between the ultra-high-resolution mass peak intensity profile of each component and the antioxidation activity profile from the 33 chromatographically separated fractions. Certain chemicals showed chromatographic distributions within several fractions, which is similar to activity change behavior. An accurate active compound mass can then be determined using ultra-high-resolution $15 \mathrm{~T}$ FT-ICR electrospray ionization mass spectrometry (UHR ESI MS), and the exact chemical formulae can be determined directly using accurate mass values and the isotopic fine structure from mass peaks [10]. Therefore, a probable candidate list with their chemical formulae can be quickly determined. Here, this list of possible antioxidants and their chemical formula from mulberry extracts were annotated by searching natural product databases to validate the updated performance of our new scoring algorithm adopted within SCAMP. In addition, two new scoring algorithms were suggested to improve the efficiency of active compound enrichment. The first method considers the summed peak intensity value, in addition to the behavioral similarity index (correlation coefficient), to account for the concentration dependence of activity. The second method resolves different components within the same mass peak by identifying that peak in multiple fractions. Therefore, certain mass peaks appear discontinuously through sequential fractions due to their different chromatographic retention properties. If certain components have different structures but the same mass, then those components will be separated chromatographically, with different retention times, and will elute into different fractions. This grouping strategy enhances the specificity of functional compound discovery using SCAMP by avoiding overestimation of correlation coefficients by using additional scores for multiple components in the same mass peak.

\section{Methods}

All MS was performed using both 15 T FT-ICR (SolariX; Bruker Daltonics, Billerica, MA, USA) and Q-TOF (SYNAPT G2-Si High Definition Mass Spectrometer; Waters
Co., Milford, MA, USA) mass spectrometers. UHR ESI/ FT-ICR MS was used to profile compounds in fractions and determine the molecular formulae of compounds; UPLC/MS was employed to identify SAOx in mulberry extracts using high-resolution mass profiling and MSMS.

\section{Sample preparation}

All standards were purchased from Chengdu Must Biotechnology and Wuhan ChemFaces Biochemical (China). Methanol (HPLC-grade), ethanol, acetonitrile, and ethyl acetate were purchased from J.T. Baker (Phillipsburg, NJ, USA). Dried fruits of Morus alba were purchased from a local herbal market in Gyeongbuk, South Korea. Fruit samples were stored at $-20^{\circ} \mathrm{C}$ immediately after collection. Fresh fruit samples $(2 \mathrm{~kg})$ of Morus alba were lyophilized at $-40^{\circ} \mathrm{C}$ (FD 8518; Ilshin Lab. Co., Ltd., Yangju, South Korea). After drying, the samples were ground and sieved through a $0.3-\mu \mathrm{m}$ mesh, and stored at $-20{ }^{\circ} \mathrm{C}$ until analysis. Dried mulberry fruit (200 g) was extracted three times using $1 \mathrm{~L}$ of $80 \%$ ethanol over 3 days at room temperature as shown in Fig. 2. This $80 \%$ ethanol extract was evaporated and then suspended in $1000 \mathrm{~mL}$ of distilled water and successively partitioned three times with the same volume of ethyl acetate to yield the ethyl acetate fraction $(9.3 \mathrm{~g})$.

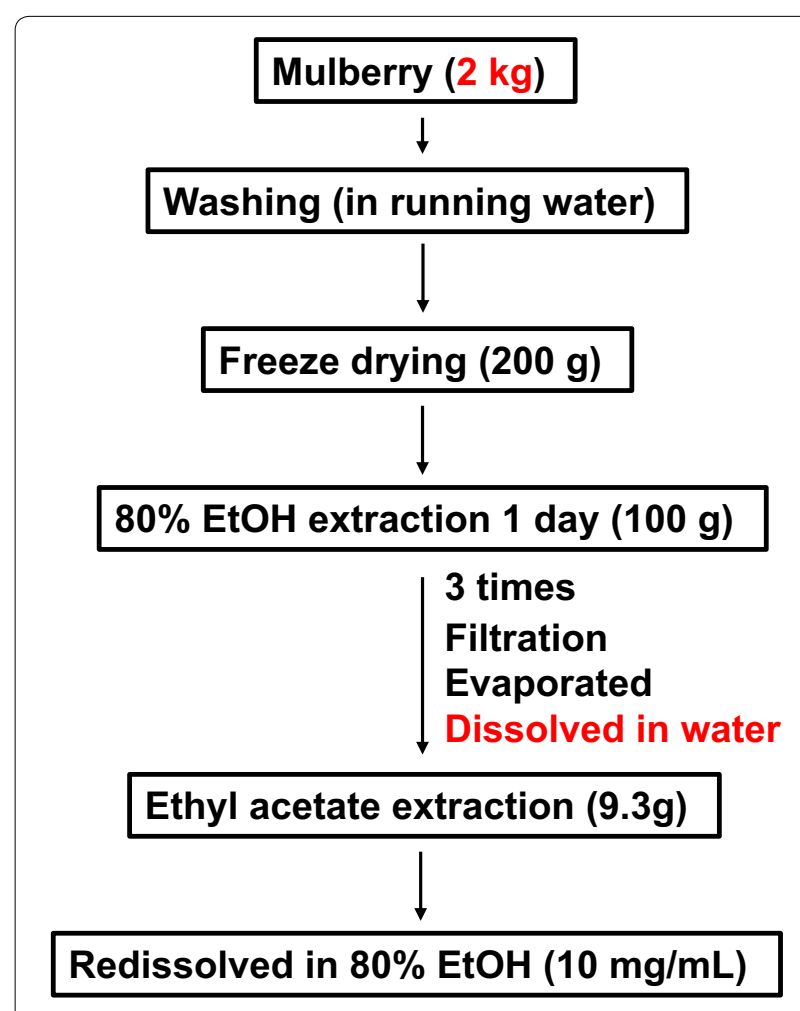

Fig. 2 Sample preparation. Dried ground mulberry fruit was extracted using $80 \%$ ethanol over 3 days and then extracted using ethyl acetate to yield the ethyl acetate fraction 


\section{UPLC/MS and MS/MS}

Samples $(5 \mu \mathrm{L})$ were prepared by diluting the ethyl acetate extract from mulberry (EAEM) to $10 \mathrm{mg} /$ $\mathrm{mL}$ with methanol. Samples were then analyzed by UPLC/Q-TOF MS. UPLC/MS analysis was performed on a Waters ACQUITY UPLC system coupled with a Waters SYNAPT G2-Si High Definition Mass Spectrometer equipped with an electrospray ionization (ESI) source (Waters Co.). Chromatographic separation was carried out using an ACQUITY UPLC HSS $\mathrm{C} 18(100 \mathrm{~mm} \times 2.1 \mathrm{~mm}, 1.8 \mu \mathrm{m})$ column at $40^{\circ} \mathrm{C}$, and an auto-sampler temperature of $10{ }^{\circ} \mathrm{C}$. Mobile phase A consisted of water, while mobile phase $B$ consisted of acetonitrile. The solvent gradient was initially $3 \% \mathrm{~B}$, changing to $3 \%-29 \% \mathrm{~B}$ over $50 \mathrm{~min}$ at a flow rate of $0.3 \mathrm{~mL} / \mathrm{min}$. This was followed by a 15 -minute washing period using $95 \% \mathrm{~B}$ prior to the next sample injection. Chromatograms were monitored at $310 \mathrm{~nm}$ using a UV detector. For the UPLC-HDMS analysis, capillary voltage and cone voltage were set at $2.0 \mathrm{kV}$ in negative ion electrospray mode. Other conditions included a source temperature of $100{ }^{\circ} \mathrm{C}$, desolvation gas flow of $900 \mathrm{~L} / \mathrm{h}$ at a temperature of $500{ }^{\circ} \mathrm{C}$, an extraction cone voltage of $4.0 \mathrm{~V}$, a collision energy of $4 \mathrm{eV}$, and a cone gas flow of $0 \mathrm{~L} / \mathrm{h}$. The scan range was $\mathrm{m} / \mathrm{z} 50-1500$. Mass scale was corrected during acquisition using an external reference (Lock-Spray), which consisted of a $300 \mathrm{ng} / \mathrm{mL}$ solution of leucine enkephalin (Sigma Aldrich) at a flow rate of $20 \mu \mathrm{L} / \mathrm{min}$; this generated a reference ion in negative ion mode $\left([\mathrm{M}-\mathrm{H}]^{-}\right.$with $\left.\mathrm{m} / \mathrm{z}=554.2615\right)$ to ensure accuracy and reproducibility. All data were acquired using MassLynx ${ }^{\mathrm{TM}}$ (V4.2) software in centroid mode.

\section{Sample fractionation (Prep LC)}

Analysis was carried out using an HPLC system (Shimadzu, Japan) equipped with an LC-20AR pump, SPD20A detector, SIL-10AP auto-sampler, and an FRC-10A fraction calculator. Each of three different amounts (300, 540 , and $1080 \mathrm{mg}$ ) of EAEM were diluted with $5 \mathrm{~mL}$ of ethanol and injected onto a C18 column $(250 \times 21.2 \mathrm{~mm}$, $7 \mu \mathrm{m}$, PrepHT 300SB; Agilent, Santa Clara, CA, USA), respectively, and separated using a binary mobile phase composed of solvents A (water) and B (acetonitrile) at a flow rate of $10.0 \mathrm{~mL} / \mathrm{min}$. During this, a solvent gradient of $3 \%$ B for $0-5 \mathrm{~min}, 3 \%-29 \%$ B for $5-50 \mathrm{~min}$, and $29 \%-$ $56 \%$ B for $50-65$ min was used. This was followed by a 15-min equilibrium period under initial conditions prior to sample injection. Chromatograms were monitored at $310 \mathrm{~nm}$ using the UV detector. Fractions were collected every $2 \mathrm{~min}$ and up to 33 fractions were collected in total. After fractionation, three sets of 33 fractions were merged into a single set of 33 fractions.

\section{UHR 15 T FT-ICR MS}

Mass profiling was performed using a 15 T FT-ICR mass spectrometer (SolariX system; Bruker Daltonics, Bremen, Germany) in negative ESI mode. Stock solutions of all 33 fractions were diluted 1000 -fold with $100 \%$ methanol and introduced into the FT-ICR mass spectrometer using direct infusion (Triversa NanoMate; Advion BioSciences, Ithaca, NY, USA) at a flow rate of $400 \mathrm{nl} / \mathrm{min}$. MS operating parameters included a capillary voltage of $2.0 \mathrm{~V}$, a mass range $(\mathrm{m} / \mathrm{z})$ from 122 to 1200 , an accumulation time of $0.7 \mathrm{~s}$, a scan number of 100 , and a sine bell apodization window function applied to the time-domain signal. External calibration was performed via quadratic regression using a $0.1 \mathrm{mg} / \mathrm{mL}$ arginine solution. All data were processed using DataAnalysis (V.4.4; Bruker Daltonics). Isotopic masses and abundances used for theoretical mass calculations were provided by the National Institute of Standards and Technology [19].

\section{Determination of DPPH radical-scavenging activity}

The DPPH radical-scavenging assay was performed in accordance with a previously described method [20], with all tests being performed in triplicate. Ascorbic acid was used as a positive control. Antioxidation activity profiles were generated by measuring the radical-scavenging activity of each fraction using 1,1-diphenyl-2-picrylhydrazyl (DPPH). Each fraction was dried with a centrifuge using a vacuum concentrator (Hanil Science Industrial, Incheon, South Korea) and reconstituted in methanol to make a $10 \mathrm{mg} / \mathrm{mL}$ stock solution. All 33 stock solutions were diluted with $50 \%$ ethanol and mixed vigorously with $100 \mu \mathrm{L}$ of $300 \mu \mathrm{M}$ aqueous DPPH solution. The absorbance of the remaining DPPH was measured at $518 \mathrm{~nm}$ after $30 \mathrm{~min}$ as follows:

$$
\%_{\text {DPPH }}=\left(\frac{A b_{t}-A b_{b}}{A b_{0}}\right) \times 100 \%
$$

In this equation, $A b_{t}=$ absorbance of the DPPH solution with tested extracts; $\mathrm{Ab}_{0}=$ the absorbance of the DPPH solution upon addition of ethanol/water (1:1, v/v); and $\mathrm{Ab}_{\mathrm{b}}=$ absorbance of tested extract solutions with the addition of $95 \%$ ethanol.

\section{Results and discussion \\ UPLC/MS and MS/MS}

UPLC/MS of the extracts was performed to confirm that the contents of standard antioxidants (SAOx) were sufficient to produce mass profiles within all 33 fractions. In LC/MS and MSMS experiments, 11 SAOx were clearly detected from EAEM (as shown in Figs. 3 and 4). Each standard anti-oxidant in the EAEM was identified using the mass values of fragment and parent ions identified by LC/MS, and LC/MSMS analyses of pure SAOx and 


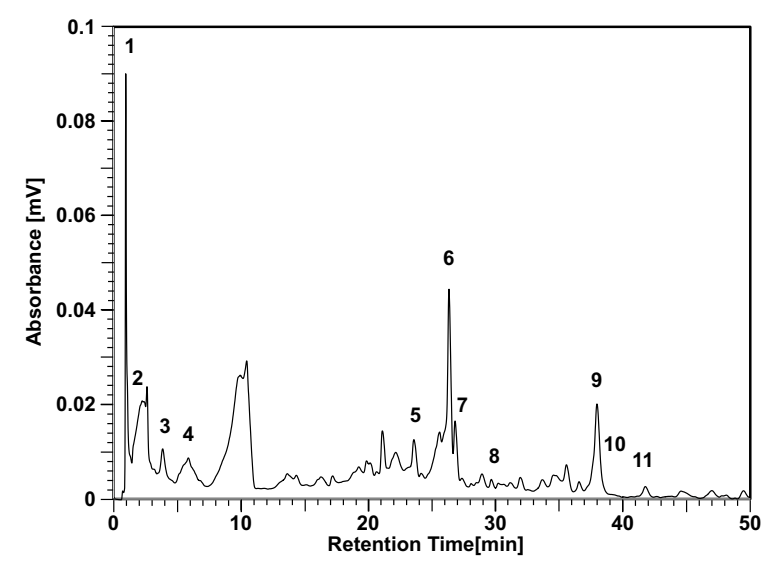

Fig. 3 UPLC/MS chromatogram of ethyl acetate extract of mulberry fruit. Ethyl acetate extracts from mulberry (EAEM) were analyzed by UPLC/Q-TOF MS and eleven SAOx were clearly detected from EAEM: (1) gallic acid, (2) gentisic acid, (3) chlorogenic acid, (4) caffeic acid, (5) dihydroquercetin, (6) rutin, (7) quercetin-3-o-glucoside, (8) quercitrin, (9) quercetin, (10) luteolin, (11) kaempferol. Each standard antioxidant in the EAEM was identified using the mass values of fragment and parent ions identified by LC/MS, and LC/MSMS analyses of pure SAOX and EAEM

EAEM as shown in Figs. 4 and 5. Quantification was performed by examination of UV absorption chromatograms and selected ion chromatograms of SAOx. The amount of SAOx used within the EAEM indicates that the sensitivity of this method is $\sim 3 \mathrm{ng}$; this means that if certain antioxidants were included in the EAEM below $\sim 3 \mathrm{ng}$, we may not detect them using current SCAMP experimental conditions. Improving the sensitivity of mass spectrometry and activity assay systems could improve detection sensitivity, but the necessary accumulation of data required for improving the signal-to-noise ratio $(\mathrm{S} / \mathrm{N})$ of mass peaks of components would be timeconsuming. Table 1 shows the approximate contents, $\mathrm{DPPH}$ anti-oxidation $\mathrm{EC}_{50}, \mathrm{MS} / \mathrm{MS}$ fragment ion list, retention time, and high-resolution mass and chemical formula of SAOx; however, the low nanogram sample used to provide the content change through the sequential fractions from preparative LC of EAEM was barely detected in our 15 T FT-ICR MS experiment. Determining antioxidation activity is critical for estimating the performance of SCAMP. Thus, the DPPH radicalscavenging activity of all 11 SAOx was measured. Each fraction was analyzed with UPLC/MS and MSMS to determine the chromatographic behavior of each SAOx within certain fraction regions. Thus, although UHR FTICR MS only produces fraction mass profiles, it is still possible to distinguish between components with identical masses but different structures. This is because the mass distribution through sequentially separated fractions shows different chromatographic behaviors and has unique distributions.

\section{Sample fractionation (Prep LC)}

Ethyl acetate extracts (EAEM) from 80\% ethanol extracts of mulberry fruit were analyzed using preparative liquid chromatography (Fig. 6). To find active compounds that exhibit behavior similar to activity profiles within sequential fractions, 33 fractions were collected sequentially every $2 \mathrm{~min}$. All SAOx were detected at similar retention times with annotated UPLC chromatograms as shown in Figs. 3 and 6. Using UPLC/MS of the fractions separated by Prep LC and the selected ion chromatogram of SAOx, we observed the large amounts of dihydroquercetin, rutin and quercetin-3-o-glucoside with trace amounts of luteolin and kaempferol as shown in Figs. 5 and 6.

The antioxidation activity profile of each fraction was generated by measuring the radical scavenging activity of all 33 fractions using 1,1-diphenyl-2-picrylhydrazyl (DPPH). The relative inhibition rates are plotted in Fig. 7. The relative antioxidation strength was then normalized by scavenging activity (Additional file 1: Table S1). SCAMP calculations were used to discover antioxidant compounds by combining all 33 mass spectra and activity data from the fractions. SCAMP performance was validated and improved by analyzing SAOx (previously reported [12-18] as anti-oxidative constituents of mulberry extract) by LC/MS. The antioxidant's radical scavenging activity was then measured using 1,1-diphenyl-2-picrylhydrazyl (DPPH) assays to provide $\mathrm{EC}_{50}$ values for all of the SAOx, given in Table 1. Eleven SAOx were observed in EAME using LC/MS and LC/MSMS, where the performance of scoring systems of the antioxidation strength of compounds in EAME was estimated by the rank of the known SAOx. If most of the SAOx were ranked high in the scoring system, then the scoring system is reliable to be applied to discover the antioxidants from plant extracts.

\section{Ultra-high-resolution mass spectra}

A UHR mass spectrum of EAME was generated using a 15 T FT-ICR MS, as shown in Fig. 8. A total of 520,000 peaks were observed over a mass-to-charge ratio $(\mathrm{m} / \mathrm{z})$ range of 120-1200, with $\mathrm{S} / \mathrm{N}>4$. We accumulated three blank mass spectra, where averaged blank spectra peaks were removed from the mass peak of each fraction to filter background peaks. The data were also reduced without loss of information using a de-isotoping procedure to reduce the computational burden. This was done by identifying ${ }^{13} \mathrm{C}$ isotopic peaks and deleting up to three heavy carbon atoms in the corresponding chemical formula. Deisotoping is carried out as follows. 


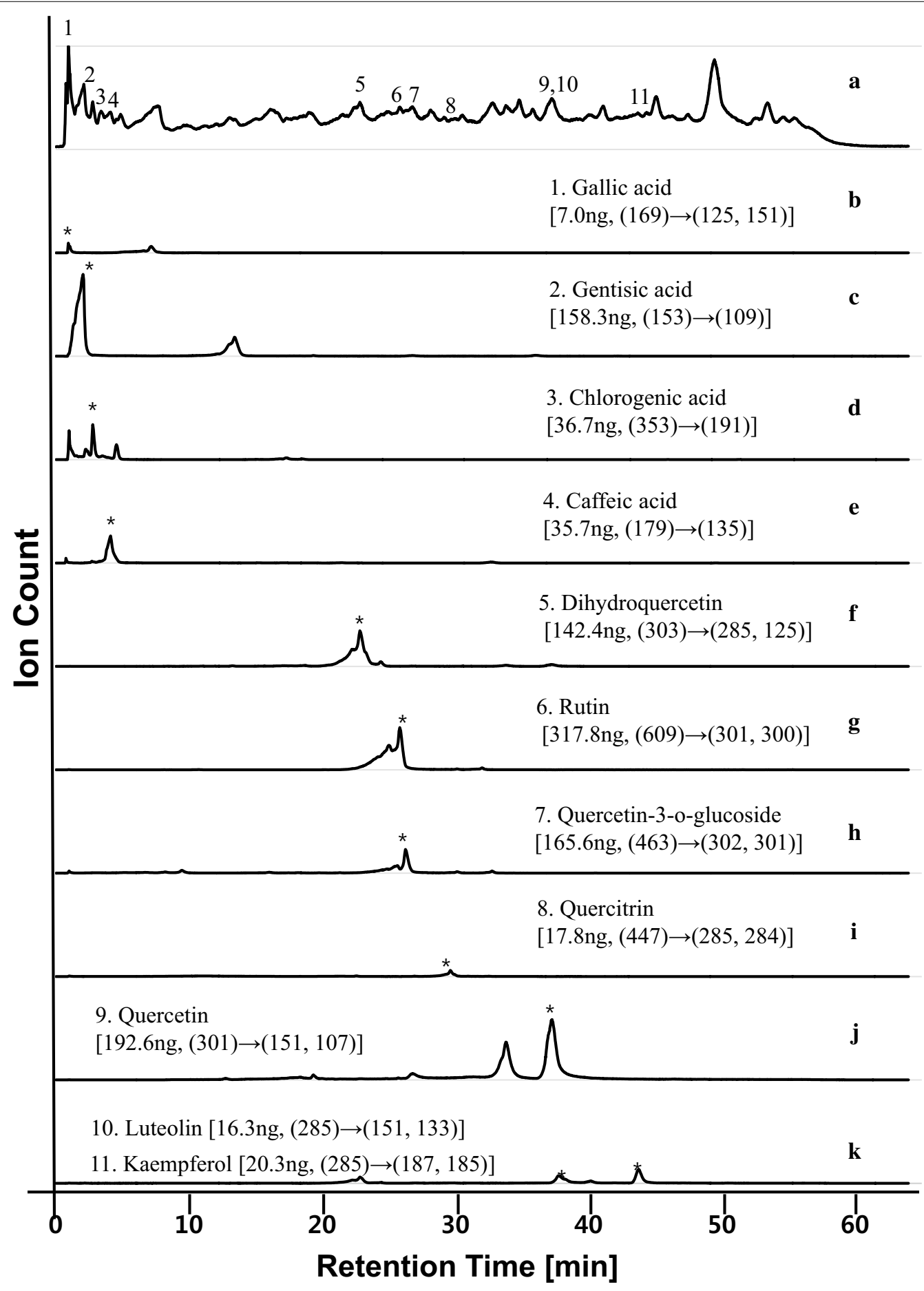

Fig. 4 Total ion chromatogram and selected ion chromatogram of EAEM.Ethyl acetate extracts from mulberry (EAEM) were analyzed by UPLC/QTOF MS and MSMS and eleven SAOx were clearly detected from EAEM. (a) Total ion chromatogram of EAEM. Selected ion chromatograms of SAOx were shown in (b) gallic acid, (c) gentisic acid, (d) chlorogenic acid, (e) caffeic acid, (f) dihydroquercetin, (g) rutin, (h) quercetin-3-o-glucoside, (i) quercitrin, (j) quercetin, and (k) luteolin and kaempferol. Each standard anti-oxidant in the EAEM was identified using the mass values of parent and fragment ions by LC/MS and LC/MSMS of pure SAOX and EAEM 


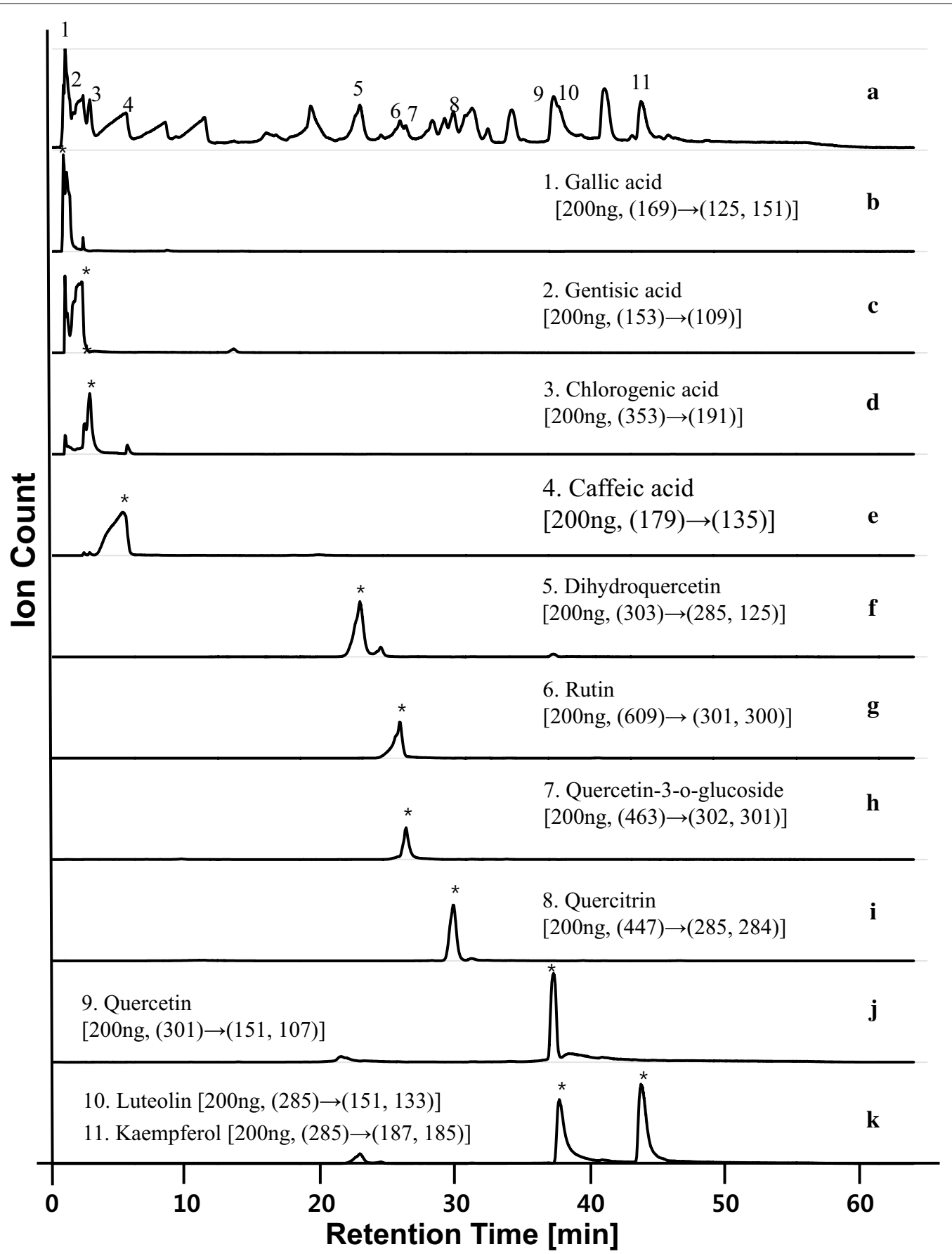

Fig. 5 Total ion chromatogram and selected ion chromatogram of standard antioxidant mixture. A standard antioxidant mixture was analyzed by UPLC/Q-TOF MS and eleven SAOx were clearly detected from the SAOx mixture : (a) total ion chromatogram, (b) gallic acid, (c) gentisic acid, (d) chlorogenic acid, (e) caffeic acid, (f) dihydroquercetin, (g) rutin, (h) quercetin-3-o-glucoside, (i) quercitrin, (j) quercetin, and (k) luteolin and kaempferol. Each standard anti-oxidant in the SAOx mixture was identified using the mass values of fragment and parent ions identified by LC/MS, and LC/ MSMS analyses of pure SAOx mixture

The peaks were sorted by the intensity in the descending order. Then starting from the highest intensity peak, the existence of $\mathrm{C}^{13}$ or $2 \mathrm{C}^{13}$ isotope peaks was tested by comparing the calculated and observed mass values and those isotopic peaks were removed from the candidate list if existed. Scripts for calculating deisotoping 
Park et al. Plant Methods (2017) 13:108

Page 8 of 16

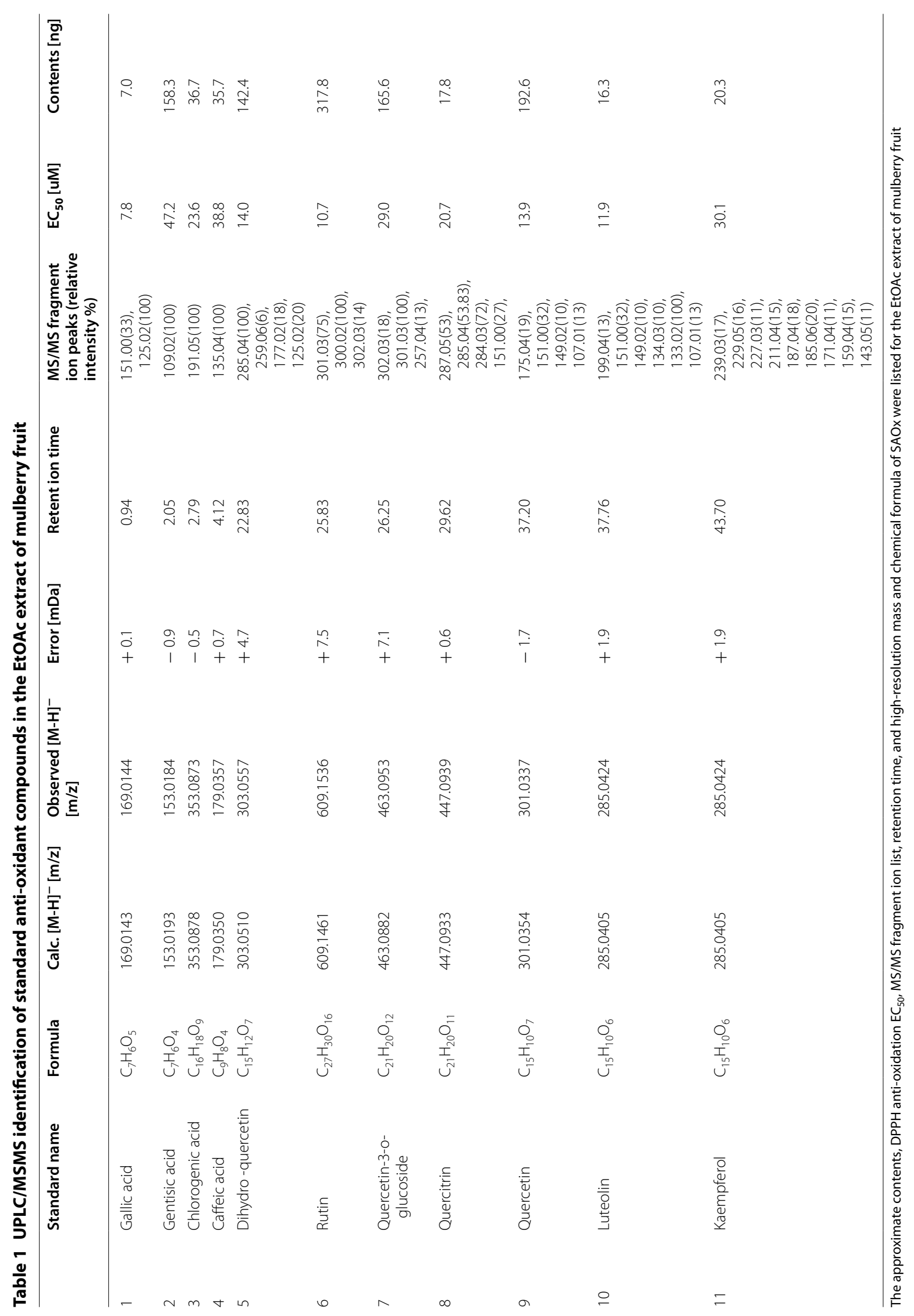




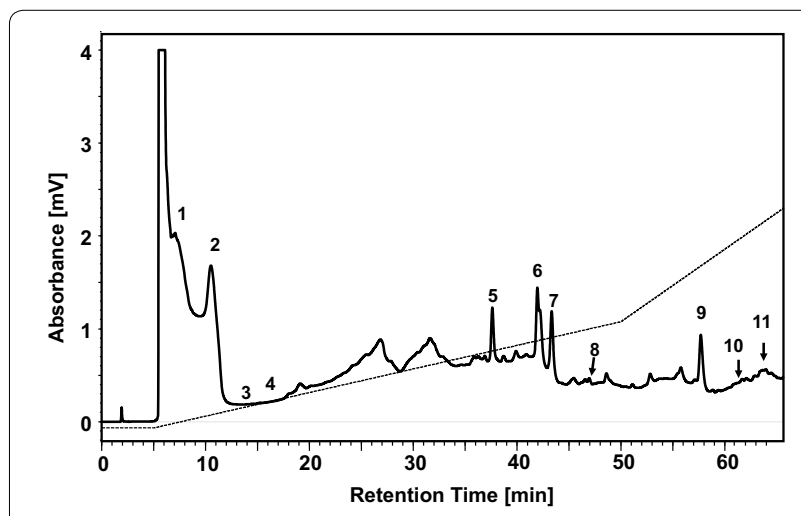

Fig. 6 Preparative HPLC chromatogram of Morus alba L. EtOAC extract. Ethyl acetate extracts (EAEM) from $80 \%$ ethanol extracts of mulberry fruit were analyzed using preparative liquid chromatography and all SAOx were detected at similar retention times with annotated UPLC chromatograms as annotated with the same number inside the chromatogram

and scoring were written in PERL programming language and are available from http://zemanet.net/scamp. A UHR mass profile of the compounds in EAEM was obtained and all 11 SAOx were detected at high mass accuracy (measurement error $<0.05 \mathrm{mDa}$ ), as shown in Table 2. Almost all SAOx chemical formulae can be directly determined with accurate mass values and isotopic distributions from UHR mass spectra. Some molecules do not show strong mass spectral intensities within the total extract mixture; however, if they are separated into 33 fractions then most peak intensities become stronger due to the collection of molecules with similar physical properties within the same chromatographic fraction. UHR mass spectra from all 33 fractions were acquired three times for each fraction using a $15-\mathrm{T}$ FT-ICR MS and the average mass spectrum was used as a mass profile in SCAMP calculations.

\section{SCAMP scoring system}

A mass spectrum can be divided into multiple $\mathrm{m} / \mathrm{z}$ bins based on the resolution of the mass spectrometer used. For example, the $\mathrm{m} / \mathrm{z}$ range from 120 to 1200 can be divided into 1081 bins with unit resolution. A mass profile of the jth $\mathrm{m} / \mathrm{z}$ bin can be represented by the vector $M$, where $M_{j}=\left\{m_{1 j}, m_{2 j}, m_{3 j}, \ldots, m_{N j} ; N=\right.$ fraction number\}. In this equation, $\mathrm{m}_{\mathrm{kj}}=$ the mass peak intensity of the jth $\mathrm{m} / \mathrm{z}$ bin of fraction $\mathrm{k}$, and $\mathrm{N}$ is the total number of fractions. If there is more than one peak within an $\mathrm{m} / \mathrm{z}$ bin, their intensities are summed to assign $m_{\mathrm{kj}}$. In the same way, the activity profile vector is defined as $A=\left\{a_{1}\right.$, $\left.a_{2}, a_{3}, \ldots, a_{N}\right\}$, where $a_{k}$ is the activity of fraction $k$. The original SCAMP algorithm only considered the similarity between mass intensity and activity changes through all fractions. The first previous scoring method [5] summed the product of the normalized mass intensity vector $\left(\mathrm{M}_{\mathrm{j}}^{0}=\left\{\mathrm{m}_{1 \mathrm{j}}^{0}, \mathrm{~m}_{2 \mathrm{j}}^{0}, \mathrm{~m}_{3 \mathrm{j}}^{0}, \ldots, \mathrm{m}_{\mathrm{Nj}}^{0} ; \mathrm{N}=\right.\right.$ fraction number $\left.\}\right)$,
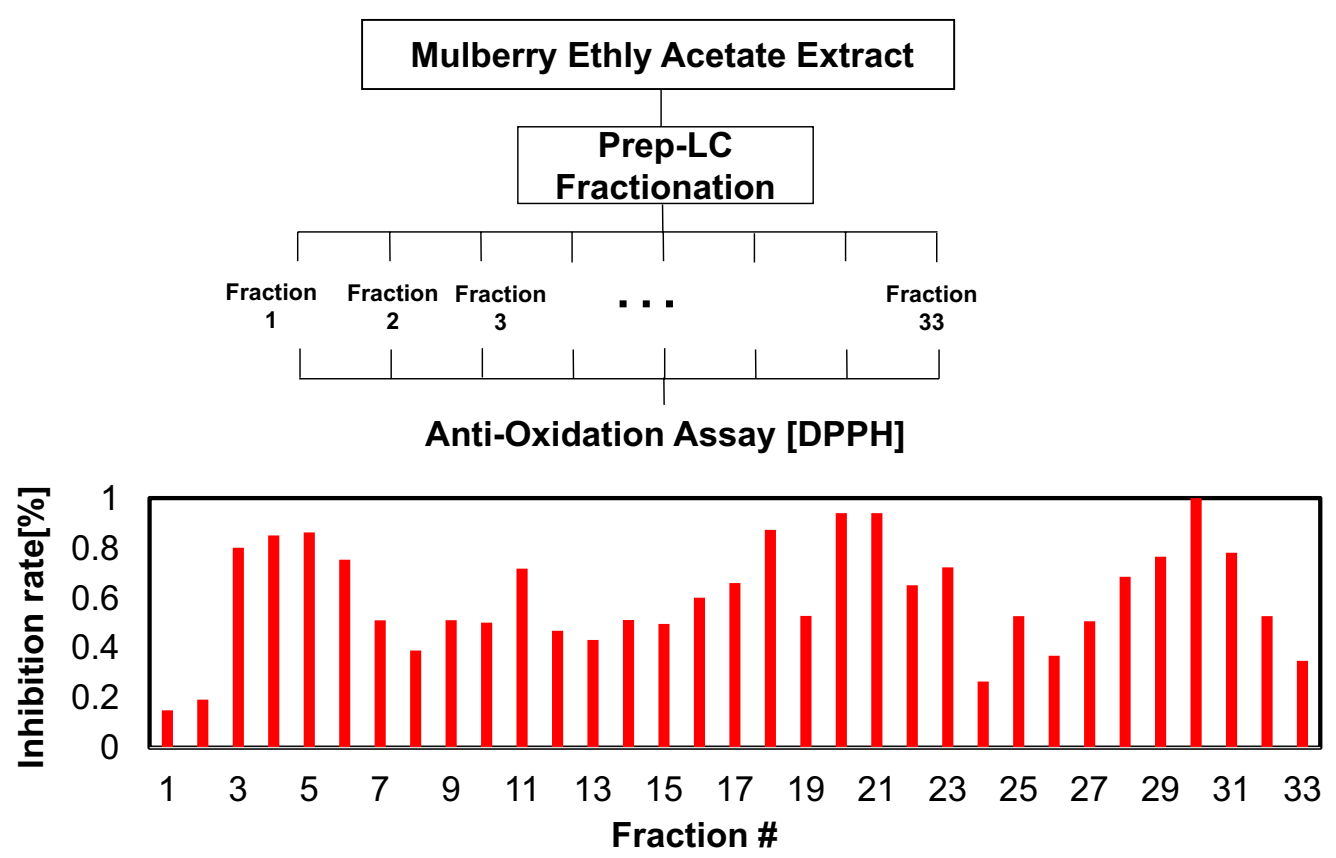

Fig. 7 Fractionation and Activity data generation. Ethyl acetate extracts (EAEM) from mulberry fruit were analyzed using preparative liquid chromatography and sequential fractions, 33 fractions were collected sequentially every 2 min. The antioxidation activity profile of each fraction was generated by measuring the radical scavenging activity of all 33 fractions using 1,1-diphenyl-2-picrylhydrazyl (DPPH) 


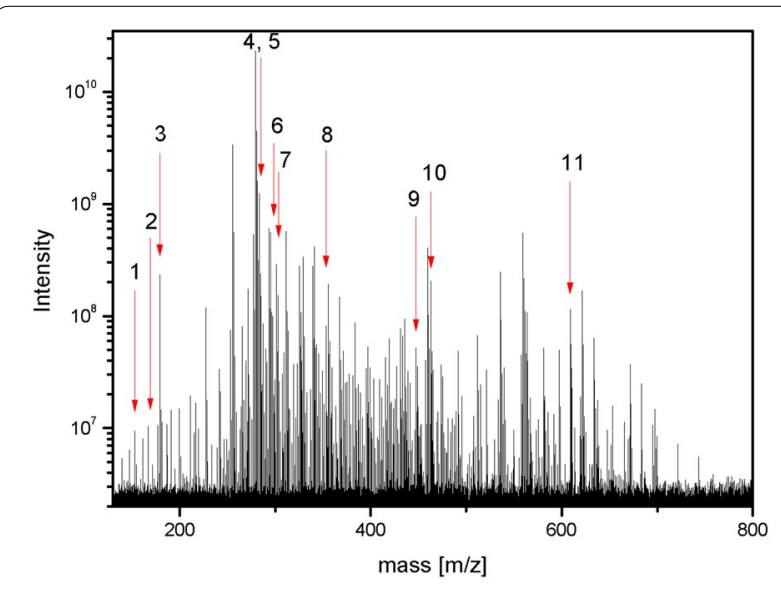

Fig. 8 15T FT-ICR mass spectrum of the EtOAc extract of mulberry fruit. A UHR mass spectrum of EAME was generated using a $15 \mathrm{~T}$ FT-ICR MS and all $11 \mathrm{SAOx}$ were detected at high mass accuracy with measurement error $<0.05 \mathrm{mDa}$

and normalized activity vector $\left(\mathrm{A}^{0}=\left\{\mathrm{a}_{1}^{0}, \mathrm{a}_{2}^{0}, \mathrm{a}_{3}^{0}, \ldots, \mathrm{a}_{\mathrm{N}}^{0}\right)\right.$ through all 33 fractions. The activity and mass profile vectors were normalized such that $\left|\mathrm{A}^{\mathrm{o}}\right|=1$ and $\left|\mathrm{M}_{\mathrm{j}}^{\mathrm{o}}\right|=1$ prior to the calculation of correlation coefficients. Only the relative changing trend was compared, where the total amount of active components was not considered. Scoring method 1:

$$
\text { Score } 1=C_{j}=\sum_{K=1}^{N} a_{k}^{0} m_{k j}^{0}
$$

$j=$ mass bin sequence number, $k=$ fraction number, $C_{j}=$ correlation coefficient, $a_{k}^{0}=$ normalized activity, $m_{k j}^{0}=$ normalized mass intensity of the $j$ th mass peak in fraction $k$.

To reduce the noise peak, three blank mass spectra were averaged and removed from the mass spectrum of each fraction. ${ }^{13} \mathrm{C}$ isotope peaks were removed from the peak list to reduce the calculation time using the mass measurement accuracy of $15 \mathrm{~T}$ FT-ICR MS. Initially, $\sim 520,000$ peaks with a mass bin size of $0.5 \mathrm{ppm}$ were screened using de-isotoping and blank peak deletion $\left(C_{j}>33\right)$ to reduce calculation time, resulting in $\sim 8000$ peaks. Thus, the correlation coefficient $C_{j}$ indicates the relative strength of antioxidant candidates.

Thus, intensity is not considered as a component of the correlation coefficient in scoring method 1. In real situations, if the concentration of a given component is below the detection limit of the mass spectrometer, then it would not be evident in the mass spectrum regardless of its activity. In addition, if the concentration of a given antioxidant is higher in certain fractions, then the antioxidative activities of those fractions would also be higher. The amount of a given antioxidant across multiple fractions can be calculated as the sum of peak intensities at a specific mass, in addition to the behavioral similarity index (correlation coefficient $C_{j}$ ), which was used as a new factor to indicate the concentration dependence of the activity profile. To compensate for the effects of concentration on antioxidative components included in the extract, we summed antioxidant mass peak $(j)$ intensities through all fractions $(k)$ and multiplied this by the original $C_{j}$, resulting in the second improved scoring method.

Scoring method 2:

$$
\text { Score } 2=C_{j} \times I
$$

$C_{j}=$ correlation coefficient, $I=$ peak $(j)$ intensities summation through all fractions $(k)$.

The third scoring method clusters different components within the same mass peak, but having different fraction-centered chromatographic distributions within sequential fractions, as shown in Fig. 9. For example, if some components have the same mass $(169.014 \mathrm{u})$ but two different structures, as in Fig. 9a, then those two components will present two separate chromatographic distributions within different fractions, described as regions 1 in Fig. 9c, and 2 in Fig. 9b. If the correlation coefficient $C_{j}$ is calculated using scoring method 1 without a grouping process then Score 1 (44.7, Fig. 9a) will be much higher than the $C_{j}$ of gallic acid (24.94, Fig. 9c) due to the different component in the same mass peak (which is usually one $\mathrm{M}_{\mathrm{j}}$, but can represent several structural isomers). It is thus necessary to group the fractions included in different chromatographic distributions, and consider

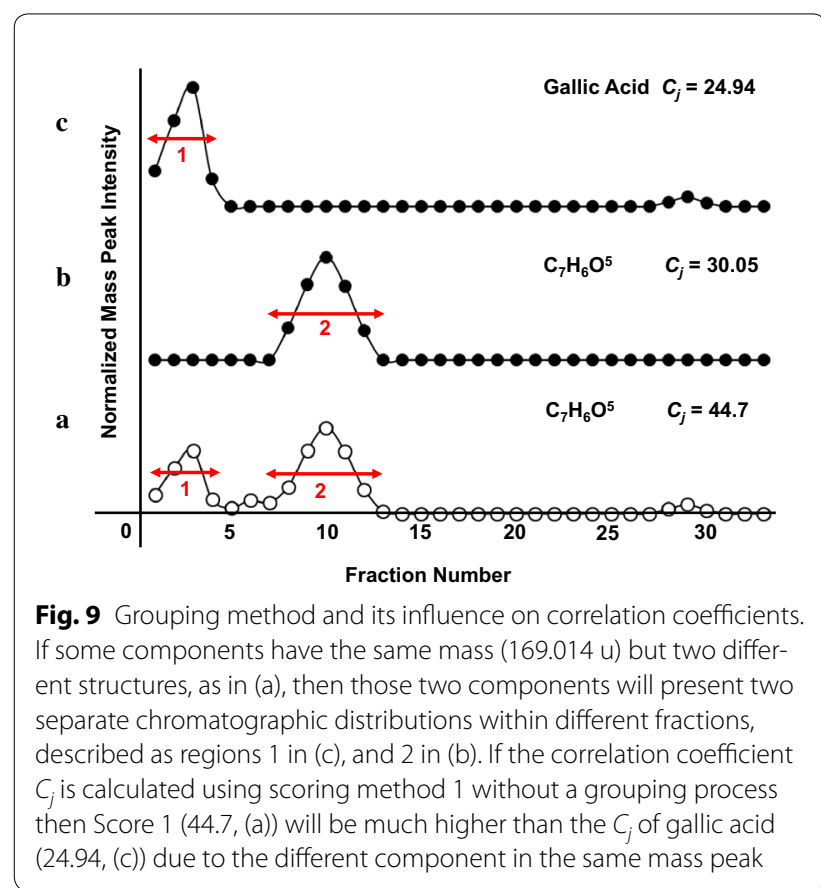


those separated groups as a new mass bin (jth $\mathrm{m} / \mathrm{z}$ bin, $\mathrm{M}_{\mathrm{j}}$ ) that is generated for different compounds. For these groupings, the mean peak intensity was first calculated using intensity values of fractions in each $\mathrm{m} / \mathrm{z}$. The algorithm then passed over data to find contiguous runs of values either less than or greater than the mean. Contiguous regions with intensity values greater than the mean became peaks, and the rest troughs. This result yielded a much lower $C_{j}$ after the grouping process, as shown in Fig. 9b, c. The grouping strategy was adopted to improve the performance of SCAMP by avoiding overestimation of the correlation coefficient by providing additional scores for multiple components within the same mass peak, as in Fig. 9.

Scoring method 3:

$$
\text { Score } 3=\frac{C_{j} \sigma^{2} I}{N_{f}^{2}}
$$

$C_{j}=$ correlation coefficient, $\sigma=$ standard deviation, $I=$ intensity summation, $N_{\mathrm{f}}=$ number of fractions in the group.

A candidate list of antioxidants in EAEM was produced using scoring method 3. Five SAOx were determined quickly in the high-score regions of the candidate list, as shown in Additional file 1: Table S1. Normalized activities for each fraction are listed in the last line of the table, and all normalized mass peak intensities are listed for each mass bin for all 33 fractions. All data were sorted by Score 3, which avoids overestimation of the score by grouping multiple components in the same mass bin. Five SAOx were observed within the top 20, indicating that we can quickly enrich active antioxidants (Table 2).

\section{Scoring algorithm estimation}

In this study, we suggest new scoring algorithms that introduce new influencing factors into the scoring system to improve the efficiency of active compound enrichment. A new concentration dependence factor for the activity profile was introduced in scoring method 2, while the third method clusters different components in the same mass peak, as in Fig. 9. SCAMP methods were validated by their enrichment factors [21-25]. Enrichment factor is defined as the ratio of the observed fraction of active compounds in the top few percent of screening to a random distribution [22]. It is widely used in virtual screening of chemical compounds for drug discovery for assessing the quality of screening protocol. Enrichment curves were obtained by plotting percent of actives found against percent of database screened and enrichment factors were calculated as the area under curve (AUC), using a plot_enrich program which is available from http://zemanet.net/plot_enrich/. Using those two new algorithms, enrichment curves were calculated to analyze the ability of different scoring methods to identify active compounds. These curves show how the fraction of active compound recovered varies with the percent compound screened. We also calculated the area under curve (AUC) to compare the scoring quantitatively. The AUC ranged from 0 to 1 , where an AUC of 1.0 indicates an ideal scoring method and an AUC of 0.5 indicates a random selection distribution. Figure 10a shows enrichment curves obtained for

Table 2 Identification of standard anti-oxidant compounds in the EtOAc extract of mulberry fruit using UHR 15-T FT-ICR mass spectrometry

\begin{tabular}{|c|c|c|c|c|c|c|}
\hline$\#$ & Standard name & Formula & Calc. $[\mathrm{M}-\mathrm{H}]^{-}[\mathrm{m} / \mathrm{z}]$ & Observed $[\mathrm{M}-\mathrm{H}]^{-}[\mathrm{m} / \mathrm{z}]$ & Error [mDa] & $\mathrm{S} / \mathrm{N}$ \\
\hline 1 & Gentisic acid & $\mathrm{C}_{7} \mathrm{H}_{6} \mathrm{O}_{4}$ & 153.01933 & 153.01933 & 0.00 & 24 \\
\hline 2 & Gallic acid & $\mathrm{C}_{7} \mathrm{H}_{6} \mathrm{O}_{5}$ & 169.01425 & 169.01426 & 0.01 & 10.1 \\
\hline 3 & Caffeic acid & $\mathrm{C}_{9} \mathrm{H}_{8} \mathrm{O}_{4}$ & 179.03498 & 179.03498 & 0.00 & 18.5 \\
\hline 4 & Luteolin & $\mathrm{C}_{15} \mathrm{H}_{10} \mathrm{O}_{6}$ & 285.04046 & 285.04047 & -0.01 & 372.3 \\
\hline 5 & Kaempferol & $\mathrm{C}_{15} \mathrm{H}_{10} \mathrm{O}_{6}$ & 285.04046 & 285.04047 & -0.01 & 372.3 \\
\hline 6 & Quercetin & $\mathrm{C}_{15} \mathrm{H}_{10} \mathrm{O}_{7}$ & 301.03538 & 301.03538 & 0.00 & 710.1 \\
\hline 7 & Dihydroquercetin & $\mathrm{C}_{15} \mathrm{H}_{12} \mathrm{O}_{7}$ & 303.05103 & 303.05104 & -0.01 & 370.6 \\
\hline 8 & Chlorogenic acid & $\mathrm{C}_{16} \mathrm{H}_{18} \mathrm{O}_{9}$ & 353.08781 & 353.08780 & 0.01 & 191.4 \\
\hline 9 & Quercitrin & $\mathrm{C}_{21} \mathrm{H}_{20} \mathrm{O}_{11}$ & 447.09329 & 447.09326 & 0.03 & 53.9 \\
\hline 10 & Quercetin-3-o-glucoside & $\mathrm{C}_{21} \mathrm{H}_{20} \mathrm{O}_{12}$ & 463.08820 & 463.08820 & 0.00 & 467.9 \\
\hline 11 & Rutin & $\mathrm{C}_{27} \mathrm{H}_{30} \mathrm{O}_{16}$ & 609.14611 & 609.14610 & 0.01 & 245.5 \\
\hline
\end{tabular}

A UHR mass profile of the compounds in EAEM was obtained and all 11 SAOx were detected at high mass accuracy with measurement error $<0.05 \mathrm{mDa}$ 


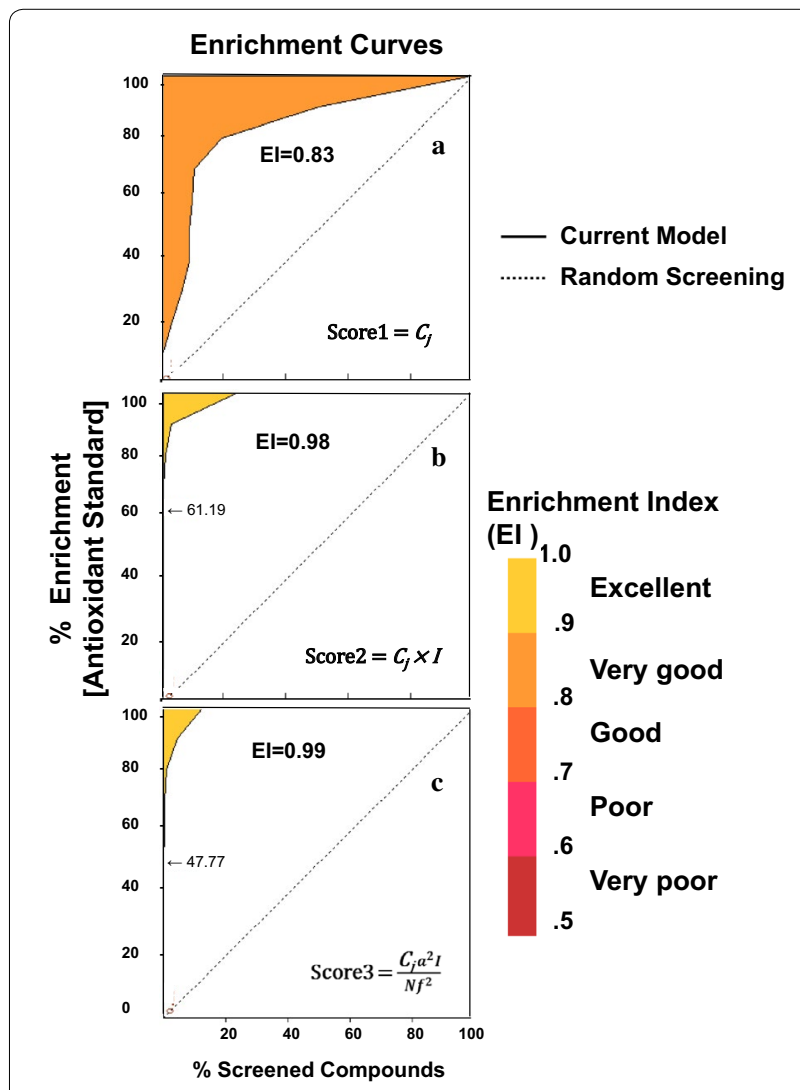

Fig. 10 Active compound enrichment curves by different scoring methods. a The antioxidative compound enrichment curve $(E I=0.83)$ of the scoring method 1 was obtained for correlation coefficients $C_{j}$ between normalized activity and mass profiles as previous SCAMP methods. b The antioxidative compound enrichment curve $(E I=0.98)$ of the scoring method 2 . To compensate for the effects of concentration on antioxidative components included in the extract, we summed antioxidant mass peak intensities through all fractions $(k)$ and multiplied this by the original $C_{j}$, resulting in the second improved scoring method. c The enrichment curve $(E I=0.99)$ for the third scoring method. If some components have the same mass but different structures, then those two components will present two separate chromatographic distributions and within different fractions. The grouping strategy was adopted to improve the performance of SCAMP by avoiding overestimation of the correlation coefficient by providing additional scores for multiple components within the same mass peak

correlation coefficients $C_{j}$ between normalized activity and mass profiles as per previous SCAMP methods. The mass peak intensities were considered to be a major influencing factor in scoring method 2 (Score 2), as shown in Fig. 10b, in which de-isotoping and blank peak deletion were applied (where $C_{j}$ values less than 30 were removed). Figure 10c shows enrichment curves for the third scoring method (Score 3) where the grouping of fractions was applied. AUC values calculated for the original $C_{j}$ value were 0.83 , while those for methods 2 and 3 were 0.98 and 0.99 , respectively. Curves obtained for the correlation coefficients $C_{j}$ (as in the previous SCAMP) deviate from the random screening line and AUC, meaning that this method detects active compounds reasonably well. The second method yielded improved enrichment plots, as confirmed by an AUC value of 0.98 . Thus, the concentration effect cannot be ignored for SCAMP calculations. Finally, a clustering strategy can improve the performance of SCAMP by considering structural isomers having the same mass. When compared to the previous method 1 , the methods 2 and 3 both greatly improved performance by the concentration factor, as confirmed by AUC values of 0.98 and 0.99 , respectively. Overall, enrichment curves and AUC values calculated for methods 1 and 2 demonstrate that method 2 performs better than method 1; however, both are better than using the correlation coefficient $C_{j}$.

Finally, a top 20 anti-oxidant candidate list was generated by sorting the mass peak list according to Score 3. The corresponding formulae are given in Table 3. Five strong standard antioxidants $[12,13,15]$ and two additional antioxidants previously found in mulberry fruits $[26,27]$ in the high-score candidate region validate the efficiency of the scoring method to enrich the antioxidants from the components in complicated plant extract mixture. The mass peak intensities of five strong standard antioxidants showed the strong correlation with complicated activity profiles through the all fractions as shown in Fig. 11. Due to a relatively polar sugar ring, chlorogenic acid (14.2 ng) was eluted at the very beginning and dihydoxy quercetin (46.2 $\mathrm{ng})$, rutin (154.0 $\mathrm{ng})$, quercetin (71.7 ng) and luteolin ( $3.5 \mathrm{ng}$ ) were eluted in the later fractions as similar eluting sequence in UPLC/MS in Fig. 3 and also observed in different working groups as shown in Additional file 2: Table S2. From a survey of natural product databases [28] and reference literatures, six of these antioxidants are found in other plants [29-31] and most of the antioxidants have structures corresponding to flavonoids. Four known compounds was listed in the top 20 list and never published as an antioxidant before. So it can be a new antioxidant candidates. And three new compounds showed the close correlation with activity profiles as shown in Fig. 12. Probably the very early eluting fractions and the last fractions will be the good starting fractions to find the new oxidative molecules from mulberry fruits.

\section{Conclusions}

Antioxidant-rich mulberry fruit extract was separated into 33 fractions and analyzed using high-resolution LC/ MS, LC/MSMS, and UHR FT-ICR MS. The performance of our antioxidative component discovery method was validated by measuring the antioxidation strength of all 33 fractions using the correlation between mass and 


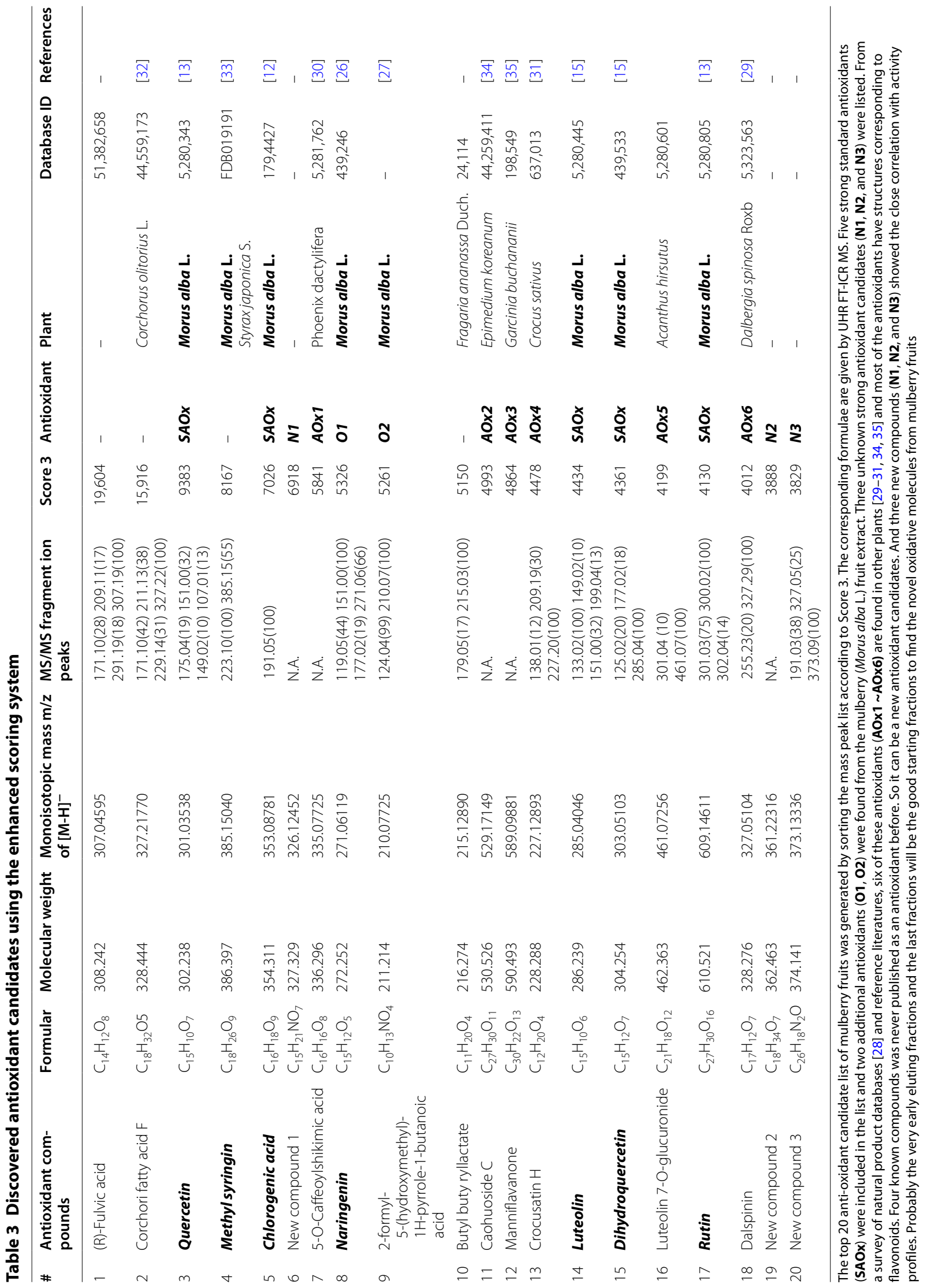




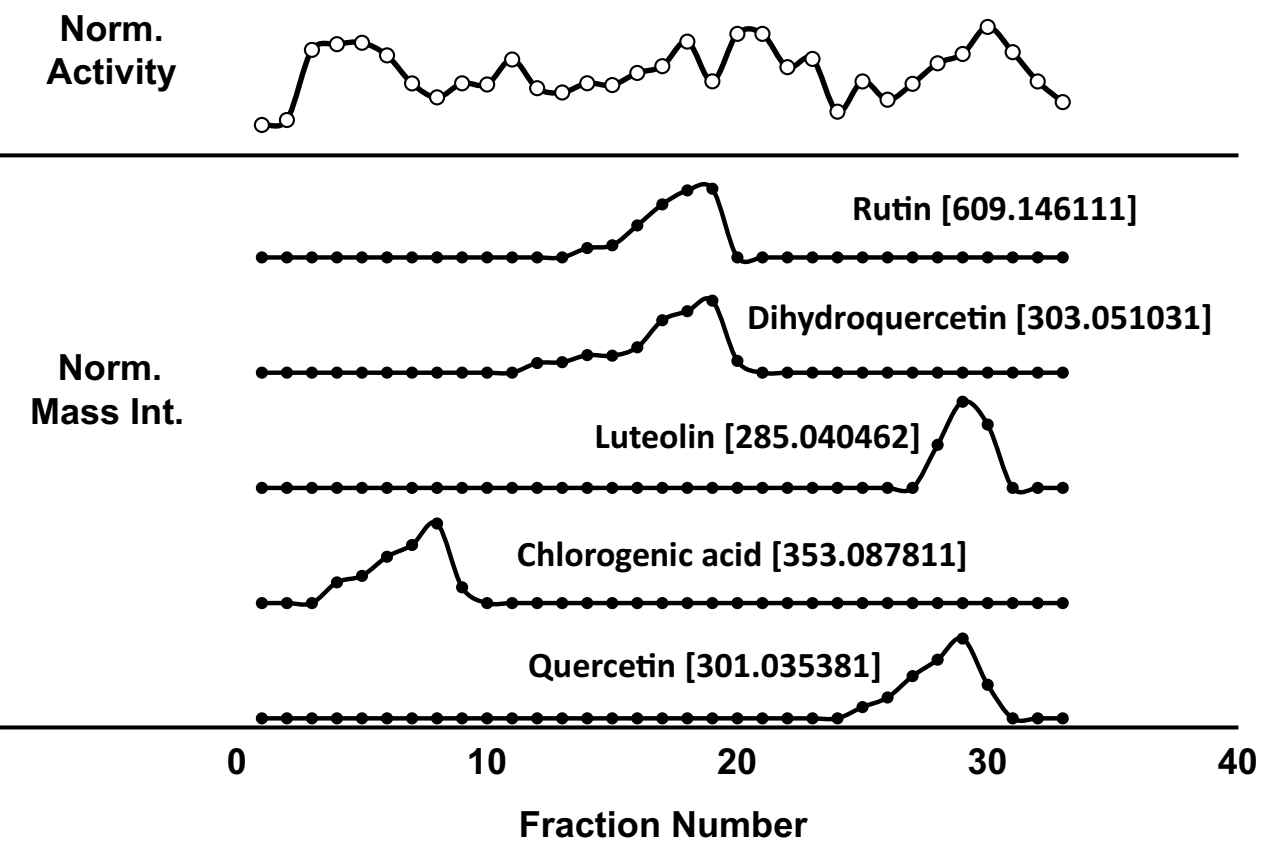

Fig. 11 Antioxidation activity and mass intensities of standard antioxidants profiles. Five strong standard antioxidants in the high-score candidate region validate the efficiency of the scoring method to enrich the antioxidants from the components in complicated plant extract mixture. The mass peak intensities of five strong standard antioxidants showed the strong correlation with complicated activity profiles through the all fractions. Due to a relatively polar sugar ring, chlorogenic acid was eluted at the very beginning and luteolin and quercetin were eluted in the later fractions as similar eluting sequence in UPLC/MS

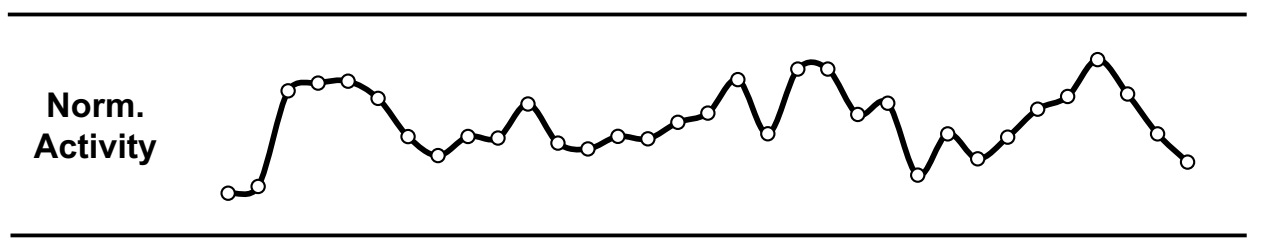

C26H18N2O [373.13336]

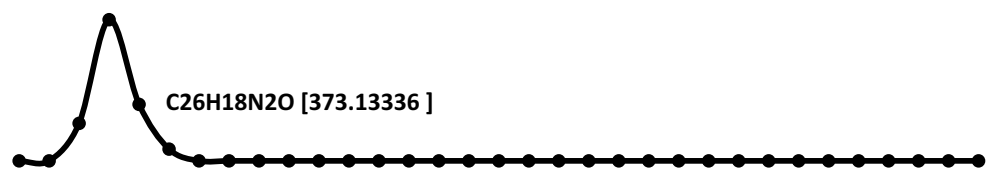

Norm.

Mass Int.

C18H3407 [361.22316]

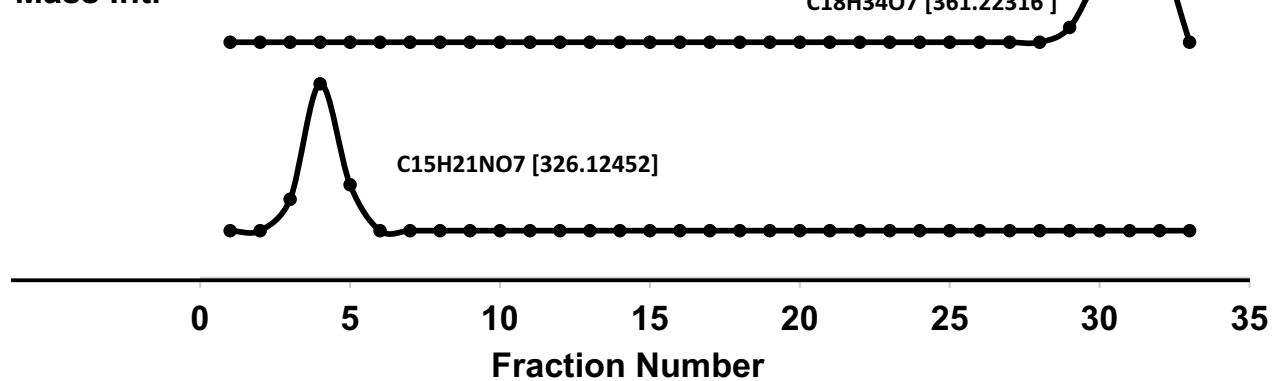

Fig. 12 Antioxidation activity and mass intensities of three new candidates. Three new compounds showed the close correlation with activity profiles and probably the very early eluting fractions and the last fractions will be the good starting fractions to find the new oxidative molecules from mulberry fruits 
activity profiles from all chromatographic fractions. Proprietary scoring algorithms were used to enrich active components and detect several strong antioxidants within the mulberry extract. The AUC was measured to compare the scoring methods quantitatively. Two new scoring systems were compared to previous scoring parameters, where the calculated AUCs of both scoring systems $(1=0.98$ and $2=0.99)$ are higher than using a previous correlation coefficient $C_{j}(0.89)$. Using an improved SCAMP enrichment system, thirteen unknown antioxidants were ranked higher than known standard antioxidants candidates in addition to known antioxidants, methyl syringin and naringenin (3.5 ng). The mass and retention time-targeted purification of unknown antioxidant candidates was shown to significantly reduce the purification time and labor involved in activity screenings.

\section{Additional files}

Additional file 1: Table S1. Anti-oxidant discovery scoring data. Normalized activities for each fraction and all normalized mass peak intensities for each mass bin for all 33 fractions are listed and sorted by the values of Score 3. Grouping information also is provided by fraction number of center position and total number of fractions in each group.

Additional file 2: Table S2. Levels of antioxidants in mulberry fruits published. Levels of anti-oxidant compounds included in mulberry fruits were measured with LC/MS by comparing the UV absorption chromatograms and selected ion chromatograms of SAOx and EAEM. Other observed levels are also compared.

\begin{abstract}
Abbreviations
EAEM: mulberry ethyl acetate extract, ethyl acetate extract of mulberry fruits; SAOx: standard antioxidants; ESI: electrospray ionization; FT-ICR MS: Fourier transform ion cyclotron resonance mass spectrometry; HPLC: high-performance liquid chromatography; HR MS: high-resolution mass spectrometry; LC: liquid chromatography; MS/MS: tandem mass spectrometry; S/N: signal-tonoise ratio; UHR: ultra-high resolution.
\end{abstract}

\section{Authors' contributions}

YJP and MRK participated in data interpretation, sample preparations, performing the fractionation with Prep-LC and DPPH experiments. SHS and SWS participated in performing the HR ESI Q-TOF MS, LC/MS, and MS/MS experiments. MSK participated in the UHR ESI FT-ICR MS experiments and data processing. HSK participated in designing experiment, developing new algorithms and preparing this manuscript. All authors read and approved the final manuscript.

\section{Author details}

${ }^{1}$ Mass Spectrometry and Advanced Instrumentation Group, Korea Basic Science Institute, Cheongju, Chungcheongbuk-do 28119, Korea. ${ }^{2}$ College of Human Ecology, Chungnam National University, Daejeon 34134, Korea. ${ }^{3}$ College of Pharmacy, Chungnam National University, Daejeon 34134, Korea. ${ }^{4}$ Department of Oriental Medicine and Biotechnology, Honam University, Gwangju 62399, Korea.

\section{Acknowledgements}

This work was supported by the National Research Foundation (NRF) of Korea grant funded by the Korea government (MSIP) (Grant No: 2014R1A2A2A04005236) and Korea Institute of Planning and Evaluation for Technology in Food, Agriculture, Forestry and Fisheries (IPET) through High
Value-added Food Technology Development Program, funded by Ministry of Agriculture, Food and Rural Affairs (MAFRA) (116035-03-2-CG000).

\section{Competing interests}

I confirm that I have read BioMed Central's guidance on competing interests and have included a statement indicating that none of the authors have any competing interests in the manuscript.

\section{Funding}

The National Research Foundation (NRF) of Korea grant funded by the Korea government (MSIP) (Grant No: 2014R1A2A2A04005236) and Korea Institute of Planning and Evaluation for Technology in Food, Agriculture, Forestry and Fisheries (IPET) through High Value-added Food Technology Development Program, funded by Ministry of Agriculture, Food and Rural Affairs (MAFRA) (116035-03-2-CG000).

\section{Publisher's Note}

Springer Nature remains neutral with regard to jurisdictional claims in published maps and institutional affiliations.

Received: 1 June 2017 Accepted: 22 November 2017

Published online: 06 December 2017

\section{References}

1. Butler MS. The role of natural product chemistry in drug discovery. J Nat Prod. 2004;67:2141-53.

2. Cragg GM, Newman DJ, Snader KM. Natural products in drug discovery and development. J Nat Prod. 1997;60:52-60.

3. Harvey AL. Natural products in drug discovery. Drug Discov Today. 2008;13:894-901.

4. Franz B, Abraham W, Martin S. Natural product isolation-how to get from biological material to pure compounds. Nat Prod Rep. 2013:30:525-45.

5. Park KH, Yoon KJ, Kwon K-H, Kim HS. High-throughput active compound discovery using correlations between activity and mass profiles. Mass Spectrom Lett. 2010;1:13-6.

6. Marshall AG, Hendrickson CL, Jackson GS. Fourier transform ion cyclotron resonance mass spectrometry: a primer. Mass Spectrom Rev. 1998;17:1-35.

7. Marshall AG. Milestones in Fourier transform ion cyclotron resonance mass spectrometry technique development. Int J Mass Spectrom. 2000;200:331-56.

8. Savory JJ, Kaiser NK, MCKenna AM, Xian F, Blakney GT, Rodgers RP, Hendrickson CL, Marshall AG. Parts-per-billion Fourier transform ion cyclotron resonance mass measurement accuracy with a "walking" calibration equation. Anal Chem. 2011;83:1732-6.

9. Kind T, Fiehn O. Metabolomic database annotations via query of elemental compositions: mass accuracy is insufficient even at less than 1 ppm. BMC Bioinformatics. 2006;7:234.

10. Miura D, Tsuji Y, Takahashi K, Wariishi H, Saito K. A strategy for the determination of the elemental composition by fourier transform ion cyclotron resonance mass spectrometry based on isotopic peak ratios. Anal Chem. 2010:82:5887-91.

11. Shi SD, Hendrickson CL, Marshall AG. Counting individual sulfur atoms in a protein by ultrahigh-resolution Fourier transform ion cyclotron resonance mass spectrometry: experimental resolution of isotopic fine structure in proteins. Proc Natl Acad Sci USA. 1998;95:11532-7.

12. Memon AA, Memon N, Luthria DL, Bhanger MI, Pitafi AA. Phenolic acids profiling and antioxidant potential of mulberry (Morus laevigata W., Morus nigra L., Morus alba L) leaves and fruits grown in Pakistan. Pol J Food Nutr Sci. 2010;60(1):25-32.

13. Wang Y, Xiang L, Wang C, Tang C, He X. Antidiabetic and antioxidant effects and phytochemicals of mulberry fruit (Morus alba L.) polyphenol enhanced extract. PLoS ONE. 2013:8(7):e71144.

14. Natić MM, Dabić DČ, Papetti A, Akšić MMF, Ognjanov V, Ljubojević M, Tešić ŽL. Analysis and characterisation of phytochemicals in mulberry 
(Morus alba L.) fruits grown in Vojvodina, North Serbia. Food Chem. 2015;171:128-36.

15. Jin Q, Yang J, Ma L, Cai J, Li J. Comparison of polyphenol profile and inhibitory activities against oxidation and a-glucosidase in mulberry (Genus Morus) Cultivars from China. J Food Sci. 2015;80(11):C2440-51.

16. Lee YJ, Kim EO, Choi SW. Isolation and identification of antioxidant polyphenolic compounds in mulberry (Morus alba L.) seeds. J Korean Soc Food Sci Nutr. 2011;40(4):517-24.

17. Pawlowska AM, Oleszek W, Braca A. Quali-quantitative analyses of flavonoids of Morus nigra L. and Morus alba L. (Moraceae) fruits. J Agric Food Chem. 2008:56:3377-80.

18. Kim JS, Ha TY, Ahn JY, Kim HK, Kim S. Composition and quantitative analysis of stilbenoids in mulberry (Morus alba L.) leaves and fruits with DAD/ UV HPLC. J Korean Soc Food Sci Nutri. 2008;37:124-8.

19. Atomic weights and isotopic compositions for all elements. National Institute of Science and Technology. http://physics.nist.gov/cgi-bin/Compositions/stand_alone.pl. Accessed 20 May 2017.

20. Granica S, Czerwińska ME, Piwowarski JP, Ziaja M, Kiss AK. Chemical composition, antioxidative and anti-inflammatory activity of extracts prepared from aerial parts of Oenothera biennis L. and Oenothera paradoxa Hudziok obtained after seeds cultivation. J Agric Food Chem. 2013;61:801-10.

21. Bender A, Glen RC. A discussion of measures of enrichment in virtual screening: comparing the information content of descriptors with increasing levels of sophistication. J Chem Inf Model. 2005;45(5):1369-75.

22. Jain AN, Nicholls A. Recommendations for evaluation of computational methods. J Comput Aided Mol Des. 2008;22(3-4):133-9.

23. Stahl $M$, Rarey $M$. Detailed analysis of scoring functions for virtual screening. J Med Chem. 2001;44(7):1035-42.

24. Mishra N, Basu A. Exploring different virtual screening strategies for acetylcholinesterase inhibitors. Biomed Res Int. 2013;2013:8-16.

25. Empereur-mot C, Guillemain H, Latouche A, Zagury J-F, Viallon V, Montes M. Predictiveness curves in virtual screening. J Cheminform. 2015;7:52-68.
26. Yang MY, Huang CN, Chan KC, Yang YS, Peng CH, Wang CJ. Mulberry leaf polyphenols possess antiatherogenesis effect via inhibiting LDL oxidation and foam cell formation. J Agric Food Chem. 2011;59(5):1985-95.

27. Kim SB, Chang BY, Jo YH, Lee SH, Han SB, Hwang BY, Kim SY, Lee MK. Macrophage activating activity of pyrrole alkaloids from Morus alba fruits. J Ethnopharmacol. 2013;145(1):393-6.

28. Natural Product Chemical Database. ZemaNet. Daejeon, Korea 2017. http://zemahealth.com:8080/np/. Accessed 1 April 2017.

29. Rajendran M, Manisankar P, Gandhidasan R, Murugesan R. Free radicals scavenging efficiency of a few naturally occurring flavonoids: a comparative study. J Agric Food Chem. 2004;52(24):7389-94.

30. Sambanthamurthi $R$, Tan Y, Sundram K, Abeywardena M, Sambandan TG, Rha C, Sinskey AJ, Subramaniam K, Leow SS, Hayes KC, Wahid MB. Oil palm vegetation liquor: a new source of phenolic bioactives. $\mathrm{Br} J$ Nutr. 2011;106:1655-63.

31. Li CY, Wu TS. Constituents of the stigmas of crocus s ativus and their tyrosinase inhibitory activity. J Nat Prod. 2002;65:1452-6.

32. Yoshikawa M, Murakami T, Shimada H, Yoshizumi S, Saka M, Yamahara J, Matsuda H. Medicinal foodstuffs. XIV. On the bioactive constituents of moroheiya. (2): New fatty acids, corchorifatty acids A, B, C, D, E, and F, from the leaves of Corchorus olitorius L. (Tiliaceae): structures and inhibitory effect on NO production in mouse peritoneal macrophages. Chem Pharm Bull. 1998;46:1008-14.

33. Kim MR, Moon HT, Lee DG, Woo ER. A new lignan glycoside from the stem bark of Styrax japonica S. et Z. Arch Pharm Res. 2007;30:425-30.

34. Li WK, Xiao PG, Liao MC, Zhag RY. Caohuoside-C from the aerial parts of epimedium koreanum nakai. Chem Res Chin Univ. 1995;2.

35. StarkTD, Germann D, Salger M, Loesch S, Balemba OB, Hofmann T. Stereochemistry, bioactivity, and taste activity of biflavanones and flavanoneC-glycosides isolated from the bark extract of Garcinia buchananii. In: Abstracts of papers of the American chemical society. Washington, DC, USA: American Chemical Society; 2014.

\section{Submit your next manuscript to BioMed Central and we will help you at every step:}

- We accept pre-submission inquiries

- Our selector tool helps you to find the most relevant journal

- We provide round the clock customer support

- Convenient online submission

- Thorough peer review

- Inclusion in PubMed and all major indexing services

- Maximum visibility for your research

Submit your manuscript at www.biomedcentral.com/submit
() Biomed Central 